\title{
Loss of p53 suppresses replication stress-induced DNA damage in ATRX-deficient neuroblastoma
}

\author{
Jesmin Akter ${ }^{1}$, Yutaka Katai ${ }^{1}$, Parvin Sultana ${ }^{1,2}$, Hisanori Takenobu ${ }^{1}$, Masayuki Haruta ${ }^{1}$, Ryuichi P. Sugino ${ }^{1}$, Kyosuke Mukae $\mathbb{D}^{1}$, \\ Shunpei Satoh ${ }^{1}$, Tomoko Wada ${ }^{1}$, Miki Ohira $\mathbb{D}^{1}{ }^{1}$, Kiyohiro Ando ${ }^{1}$ and Takehiko Kamijo (D) $^{1,2 \bowtie}$
}

(c) The Author(s) 2021

\begin{abstract}
Genetic aberrations are present in the ATRX gene in older high-risk neuroblastoma (NB) patients with very poor clinical outcomes. Its loss-of-function (LoF) facilitates the alternative lengthening of telomeres (ALT) pathway in tumor cells and is strongly linked to replication stress (RS) and DNA damage through G-quadruplex (G4) DNA secondary structures. However, limited information is available on ATRX alteration-related NB tumorigenesis. We herein knocked out (KO) ATRX in MYCN-amplified (NGP) and MYCN single copy (SK-N-AS) NB cells with wild-type (wt) and truncated TP53 at the $C$ terminus, respectively, using CRISPR/Cas9 technologies. The loss of ATRX increased DNA damage and G4 formation related to RS in TP53 wt isogenic ATRX KO NGP cells, but not in SK-N-AS clones. A gene set enrichment analysis (GSEA) showed that the gene sets related to DNA double-strand break repair, negative cell cycle regulation, the G2M checkpoint, and p53 pathway activation were enriched in NGP clones. The accumulation of DNA damage activated the ATM/CHK2/p53 pathway, leading to cell cycle arrest in NGP clones. Interestingly, ATRX loss did not induce RS related to DNA damage response (DDR) in TP53-truncated SK-N-AS cells. p53 inactivation abrogated cell cycle arrest and reduced G4 accumulation in NGP clones. The loss of p53 also induced G4 DNA helicases or Fanconi anemia group D2 protein (FANCD2) with ATRX deficiency, suggesting that ATRX maintained genome integrity and p53 deficiency attenuated RS-induced DNA damage in NB cells featuring inactivated ATRX by regulating DNA repair mechanisms and replication fork stability.
\end{abstract}

Oncogenesis (2021)10:73; https://doi.org/10.1038/s41389-021-00363-6

\section{INTRODUCTION}

Neuroblastoma (NB) is a pediatric tumor of the sympathetic nervous system that accounts for $8-10 \%$ of all childhood cancers and $15 \%$ of pediatric oncology deaths. Genomic studies revealed that patients with high-risk NB frequently harbored recurrent MYCN amplification (37\%), TERT rearrangements (23\%), and alpha thalassemia mental retardation X-linked (ATRX) mutations or deletions (11\%) [1, 2]. Mutations in ATRX were found to be mutually exclusive with TERT promoter mutations and MYCN amplification, and defined a distinct subgroup of older NB patients with poor outcomes [1-3]. In addition to human NB, loss-offunction (LoF) mutations in ATRX have frequently been detected in multiple malignancies [4-7], and have been implicated in the telomerase-independent telomere maintenance alternative lengthening of telomeres (ALT) mechanism, which has been reported in $24 \%$ of high-risk NB [8-10]. The ATRX/DAXX complex was found to be less abundant in ALT-positive NB tumors due to ATRX mutations (55\%) or low protein expression [9], which supports the relationship between the ATRX status and ALT. Previous studies described a link between the loss of ATRX, replication stress (RS), DNA damage, copy number alterations, and genomic instability [11-15]. ATRX has more recently been suggested to bind to the G-quadruplex (G4) structure [16], with its loss leading to an increase in the $\mathrm{G} 4$ structure or the formation of stable DNA:RNA hybrids (R-loops), which is considered to induce replication fork stalling and collapse and the generation of DNA double-strand breaks (DSBs) at telomeres, suggesting a role for ATRX in the resolution of the G4 structure and regulation of R-loops $[11,17]$. ATRX also forms a complex with DAXX to deposit $\mathrm{H} 3.3$, which prevents the formation of the G4 structure $[11,18,19]$ or R-loops [17], thereby maintaining fork stability during acute RS.

A recent study demonstrated that somatic mutations in TP53 pathway genes were significantly enriched in ALT-positive tumors [9]. Furthermore, p53 pathway aberrations were frequently detected in ALT NB cell lines [20]. In ALT-positive tumors, ATRX mutations are commonly connected to mutations in the tumor suppressor gene TP53 [4, 21, 22], and the LoF of p53 activates the ALT pathway [20]. Collectively, these findings highlight the involvement of both ATRX mutations and p53 pathway aberrations in NB tumorigenesis in terms of ALT. In mouse neural progenitor cells (mNPCs), ATRX deficiency promoted p53-dependent apoptosis through the accumulation of DNA damage in the embryonic brain caused by DNA RS [15]. However, DNA damage accumulation and cell death were effectively rescued in ATRX/p53 double mutant mice [23]. Although the loss of p53 promotes the growth of emergent cancer cells by reducing RS-induced DNA damage [24], the mechanisms by which p53 deficiency suppresses RS in terms of ATRX loss remain unknown.

To avoid RS, G4 helicases prevent G4-induced genome instability by resolving $\mathrm{G} 4$ structures $[25,26]$. Another Fanconi

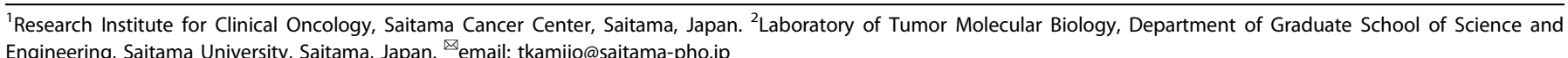

Received: 31 May 2021 Revised: 3 October 2021 Accepted: 11 October 2021

Published online: 06 November 2021 
anemia (FA) pathway protein, FANCD2, plays a key role in limiting RS by controlling the stability of stalled replication forks in cells or tumors lacking BRAC1/2 [27, 28], and has been shown to cooperate with ATRX to limit RS and promote the homologous recombination (HR)-dependent repair of DSBs [29]. In addition to the H3.3 chaperone activity of ATRX/DAXX, the histone $\mathrm{H} 3$ deposition activity of FANCD2 was necessary for protecting stalled replication forks [29]. Previous studies also revealed the transcriptional regulation of G4 DNA helicases or FA pathway genes by p53, which are involved in telomere maintenance, DNA repair, and the centromere structure [30-32]. However, the impact of this regulation in ATRX-deficient cells remains unclear.

In the present study, we revealed that ATRX depletion in TP53 wild-type (wt) NB cells was associated with an increased frequency of DSBs and a subsequent RS-induced DNA damage response (DDR), which was impaired by the loss of p53 through the activation of G4 DNA helicases or the FA DNA repair pathway protein, FANCD2. Collectively, the present results indicate that p53 deficiency limits ATRX loss-induced RS/genome integrity in NB cells by regulating DNA repair mechanisms and replication fork stability.

\section{MATERIALS AND METHODS \\ Cell lines}

Human NB cell lines (NGP, NB-69 and SK-N-AS) were obtained from official cell banks (RIKEN Cell Bank, Tsukuba, Japan and ATCC, Manassas, VA, USA). Additional details of cell lines are in the Supplementary Materials and Methods.

\section{ATRX CRISPR genome editing}

CRISPR/Cas9 technology was used to generate ATRX KO cells. We designed three guide RNAs (gRNAs) against exons 4 and 5 of ATRX (Supplementary Fig. 1A, B). Further details are presented in the Supplementary Materials and Methods.

\section{Supplementary information}

Other materials and methods and Supplementary Tables S1 to S4 are described in Supplementary Information.

\section{RESULTS}

Generation of ATRX KO cells by CRISPR/Cas9 genome editing We performed genome editing with the CRISPR/Cas9 system to recapitulate the cellular and molecular perspectives of ATRX deficiency in human NB using the NGP, NB-69, and SK-N-AS cell lines, which are wt for the gene. NGP and NB-69 cells have wt TP53 with MYCN amplifications and MYCN single copy, respectively; the MYCN single copy SK-N-AS cell line carries a TP53 truncation at its $\mathrm{C}$ terminus [33]. All three cell lines were selected for ATRX gene editing because neither displayed the ALT phenotype [8]; therefore, they were good comparable models for examining ATRX functions because ATRX is often co-mutated with TP53 in different tumors $[8,12,20]$. Our KO cells in three cell lines were characterized in detail (Supplementary Fig. 1, "Materials and methods"). As a control, we used bulked cells (referred to as Ctrl) and two clonal cell lines (referred to as Ctrl-1 and Ctrl-2) overexpressing Cas9 alone for NGP and NB-69 or SK-N-AS cells, respectively. We isolated four independent cell clones for NGP and three clones for both NB-69 and SK-N-AS with sequenceconfirmed frameshift mutations in ATRX (Supplementary Fig. 1C) and completely devoid of ATRX protein expression, except for KO (C-3) NGP cells (Fig. 1A, Supplementary Figs. 2A, B, 3A and Fig. 4A). ATRX protein expression in ATRX KO cells for NGP and SK-N-AS were also verified using immunofluorescence (IF) (Supplementary Fig. 2A, B). Therefore, we successfully established several ATRX KO isogenic cells in TP53 wt NGP, NB-69, and TP53 truncated SK-N-AS cell lines for further study.
ATRX loss induces G4 formation and RS in TP53 wt NB cells To clarify the biological consequences of ATRX deficiency, we initially examined the viability and clonogenic survival of ATRX KO NGP and NB-69 cells. An assessment of cell viability using the WST8 assay showed that ATRX loss resulted in failed cell proliferation (Fig. 1A, Supplementary Fig. 3A), which is consistent with previous findings on ATRX deficiency in mNPCs and glioblastoma cells $[12,13]$. We also observed lower clonogenic survival in ATRX KO than in Ctrl cells (Fig. 1B, Supplementary Fig. 3B).

ATRX plays a key role in the regulation of DNA replication and DNA damage repair pathways $[13,14,34]$. To ascertain whether ATRX depletion results in the accumulation of DNA damage at telomeres, we investigated $\mathrm{\gamma H} 2 \mathrm{AX}$ levels as a marker of stalled replication forks and DSBs. $\mathrm{YH} 2 \mathrm{AX}$ levels were elevated in ATRX KO cells (Fig. 1C, Supplementary Fig. 3C), suggesting increases in stalled and collapsed replication forks. We also found enhanced telomeric DDR in ATRX KO NGP cells, as indicated by the increased formation of $\mathrm{YH} 2 \mathrm{AX}$-associated telomere dysfunction-induced foci, TIF (Fig. 1D, E). This result suggests that ATRX also functions to protect against telomere DNA damage for telomere maintenance.

We then investigated whether increased levels of DNA damage under ATRX-deficient conditions were induced by the formation of G4. Consistent with previous findings [11, 35], G4 formation at stalled replication forks was associated with RS and DDR. Using a monoclonal antibody that recognizes the G4 structure in situ (1H6), the nuclear accumulation of $\mathrm{G} 4$ was found to be higher in ATRX KO cells than in Ctrl cells (Fig. 1F, G and Supplementary Fig. 3D-E). Moreover, G4s more extensively colocalized with DNA damage foci compared with telomere region in the setting of ATRX deficiency (Fig. 1F, H and Supplementary Fig. 3D). The specificity of $1 \mathrm{H} 6$ antibodies for the G4 structure has already been confirmed [11]. These results suggest that ATRX deficiency induces G4 formation, indicating a role for ATRX in resolving the G4 structure at stalled replication forks.

To clarify whether increased G4 levels result in RS in ATRX KO NGP cells, we performed a Western blot analysis of RS signaling pathways. As shown in Fig. 1l, ATRX loss increased phospho-KAP1, phospho-Chk1, and phospho-RPA32 levels. Furthermore, IF showed that ATRX loss induced phospho-RPA32 foci (Supplementary Fig. 4A) and colocalization with G4 signal (Supplementary Fig. $4 B)$, suggesting that ATRX is required to limit RS. Collectively, these results indicate that ATRX deficiency promoted RS and DDR in TP53 wt NB cells.

Recent studies reported that ATRX deficiency promote the ALT phenotype by inducing $\mathrm{HR}$, which is exhibited by tumors harboring ATRX mutations $[13,19]$. To assess the effects of ATRX loss on ALT in NB cell lines, we examined ALT-associated features in ATRX KO NGP (Supplementary Fig. 5A-D) and NB-69 cells (data not shown). The hallmarks of ALT, namely, C-circles (Supplementary Fig. 5B) and ALT-associated PML bodies (APBs) (Supplementary Fig. 5C, D), were not induced after the loss of ATRX in these KO cells. Moreover, overall telomeric DNA between Ctrl and ATRX KO cells was unchanged (Supplementary Fig. 5A). Therefore, ATRX deficiency itself was not associated with the ALT phenotype in ATRX KO TP53 wt NB cells, which is consistent with previous findings.

\section{Increased DNA damage induces the ATM/CHK2/p53 pathway in TP53 wt ATRX KO NB cells}

To elucidate ATRX-dependent transcriptional alterations and their functional consequences, we performed a microarray analysis of Ctrl and ATRX KO NGP cells. According to GSEA, upregulated genes were implicated in DSBs and HR repair, cell cycle checkpoint activation, negative cell cycle regulation, and p53 pathway activation (Fig. 2A-E). This result suggested that the pathways involved in negative cell cycle regulation and DNA damageinduced p53 pathway activation were enhanced by ATRX deficiency in TP53 wt NGP cells accompanied by transcriptional changes in their related gene members. 


\section{A.}

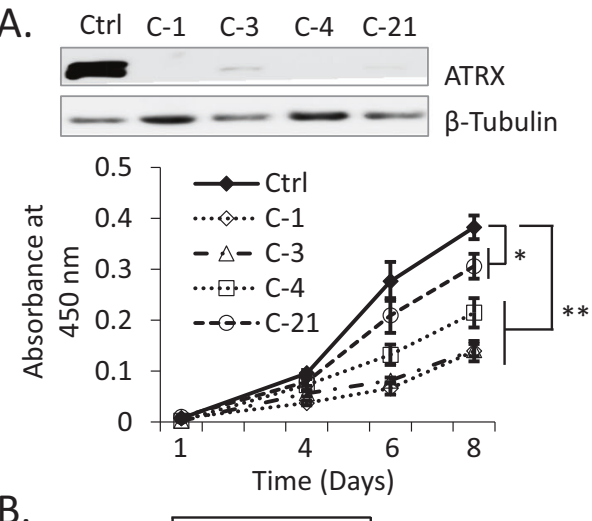

B.

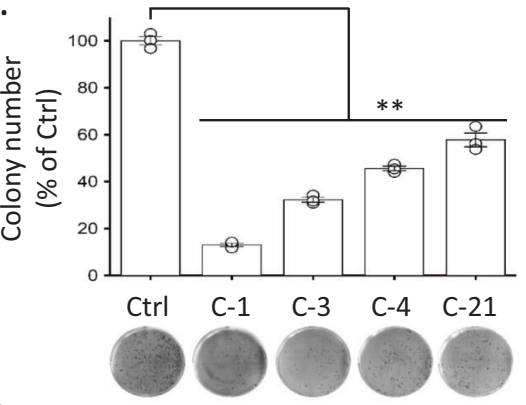

F.

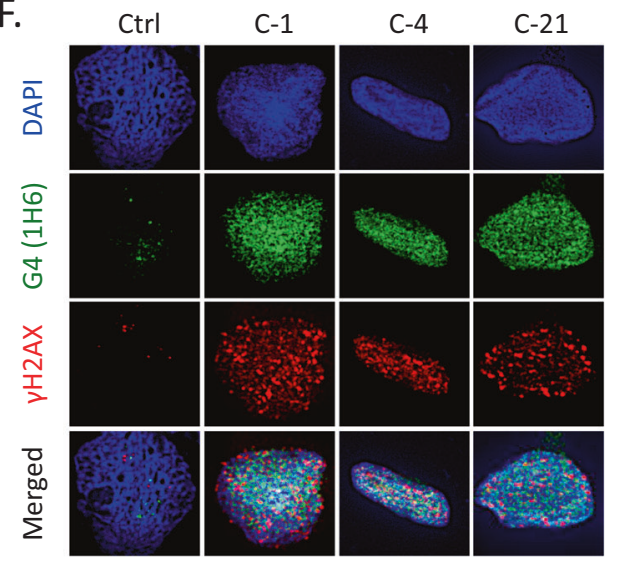

G.

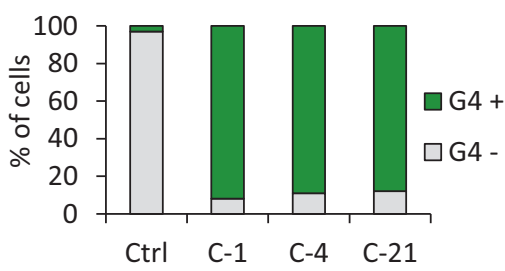

C. Ctrl C-1 C-4 C-21

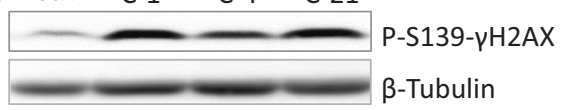

D.

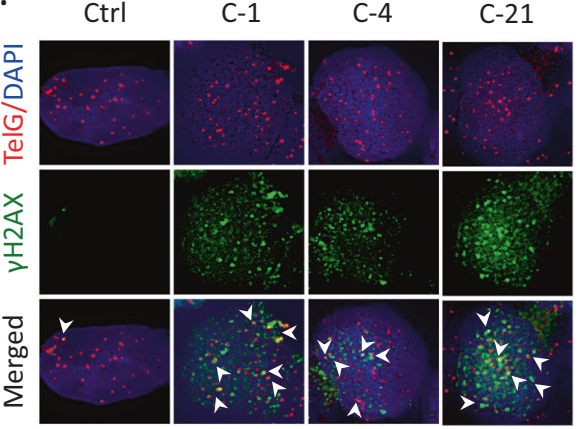

E.

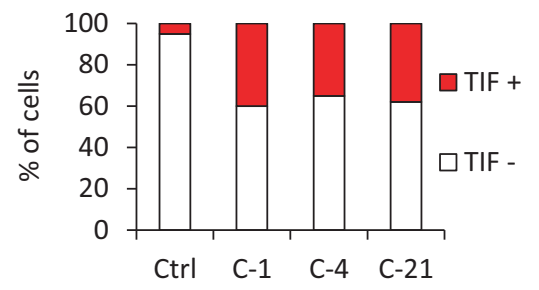

H.

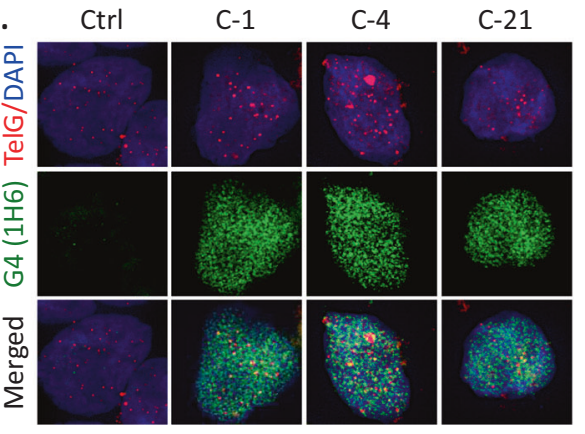

I.

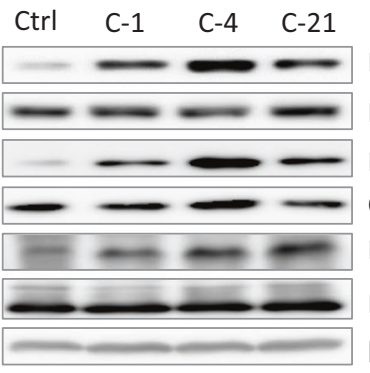

P-S824-KAP1

KAP1

P-S345-Chk1

Chk1

P-S33-RPA32

RPA32

$\beta$-Tubulin

Fig. 1 ATRX loss induces G4 formation and RS in TP53 wt NGP cells. A Western blots show the depletion of ATRX protein expression in cell lysates prepared from Cas9 control (Ctrl) and ATRX KO (C-1, C-3, C-4, and C-21) NGP cells. $\beta$-Tubulin was used as a loading control. Lower panel, growth curves show that viability was lower in ATRX KO NGP cells than in Ctrl cells. Data are expressed as means \pm standard deviation (SD), $N=3$. A two-way ANOVA followed by a multiple comparison Bonferroni post hoc test was used to compare differences between groups $\left({ }^{*} p<\right.$ 0.05 and $\left.{ }^{* *} p<0.01\right)$. B Clonogenic assay of Ctrl and ATRX KO NGP cells demonstrating the weaker proliferative abilities of KO cells than Ctrl cells. Lower panel, representative images for clonogenic formation are shown. Error bars represent SD from three technical replicates. ${ }^{* *} p<$ 0.01; A one-way ANOVA with Dunnett's and Tukey's test were used for statistical analyses. C Immunoblot showing activation of the DDR upon the depletion of ATRX, including the phosphorylation of histone H2AX on Ser-139 $(\gamma \mathrm{H} 2 \mathrm{AX})$. D, E $\gamma \mathrm{H} 2 \mathrm{AX} / \mathrm{TelG}$ immuno-FISH (D) shows increased TIF (telomere dysfunction-induced foci) in ATRX KO NGP cells. Arrows denote the colocalization of telomeric foci (red) and $\gamma \mathrm{H} 2 \mathrm{AX}$ signals (green). Cells were also stained with DAPI to visualize nuclei (blue). E Quantification of TIF+ cells among 100 cells analyzed in (D). F, G Coimmunofluorescence staining of ATRX-intact (Ctrl) and ATRX KO NGP cells with the anti-G-quadruplex (G4) antibody, 1H6 and anti$\gamma \mathrm{H} 2 \mathrm{AX}$. G Quantification of G4+ cells among 100 cells analyzed in (F). H G4 (1H6)/TelG immuno-FISH reveals colocalization of G4 on telomeric region in ATRX KO NGP cells. Nuclei are counterstained with DAPI (blue). I Representative immunoblot analysis of p-KAP1 (Ser-824), KAP1, p-Chk1 (Ser-345), Chk1, p-RPA32 (Ser-33), and RPA32, showing the activation of RS arising from ATRX deficiency. $\beta$-Tubulin served as a loading control. 
A.

GSEA Hallmark Gene sets

\begin{tabular}{|c|c|c|}
\hline \multicolumn{3}{|c|}{$\begin{array}{l}\text { Double strand break and homologous recombination } \\
\text { repair }\end{array}$} \\
\hline Name & $\begin{array}{l}\text { NOM p- } \\
\text { value }\end{array}$ & NES \\
\hline GO_RECOMBINATIONAL_REPAIR & 0.0 & 2.06 \\
\hline GO_DOUBLE_STRAND_BREAK_REPAIR & 0.0 & 1.99 \\
\hline KEGG_HOMOLOGOUS_RECOMBINATION & 0.0 & 1.86 \\
\hline DOUBLE_STRAND_BREAK_REPAIR & 0.0 & 1.73 \\
\hline \multicolumn{3}{|l|}{ G2M checkpoint activation } \\
\hline GO_REGULATION_OF_CELL_CYCLE_CHECKPOINT & 0.0 & 2.12 \\
\hline HALLMARK_G2M_CHECKPOINT & 0.0 & 1.82 \\
\hline \multicolumn{3}{|l|}{ Negative regulation of cell cycle } \\
\hline GO_NEGATIVE_REGULATION_OF_CELL_CYCLE & 0.0 & 1.88 \\
\hline $\begin{array}{l}\text { GO_NEGATIVE_REGULATION_OF_CELL_CYCLE_PR } \\
\text { OCESS }\end{array}$ & 0.0 & 1.83 \\
\hline $\begin{array}{l}\text { GO_NEGATIVE_REGULATION_OF_MEIOTIC_CELLC } \\
\text { YCLE }\end{array}$ & 0.0 & 1.98 \\
\hline \multicolumn{3}{|l|}{ p53 pathway activation } \\
\hline P53_DN.V2_DN & 0.0 & 1.90 \\
\hline PID_P53_REGULATION_PATHWAY & 0.004 & 1.76 \\
\hline PID_P53_DOWNSTREAM_PATHWAY & 0.0 & 1.73 \\
\hline TANG_SENESCENCE_TP53_TARGETS_DN & 0.0 & 1.72 \\
\hline $\begin{array}{l}\text { REACTOME_P53_DEPENDENT_G1_DNA_DAMAGE_ } \\
\text { RESPONSE }\end{array}$ & 0.011 & 1.70 \\
\hline HALLMARK_P53_PATHWAY & 0.004 & 1.58 \\
\hline BIOCARTA_P53_PATHWAY & 0.017 & 1.56 \\
\hline
\end{tabular}

F.

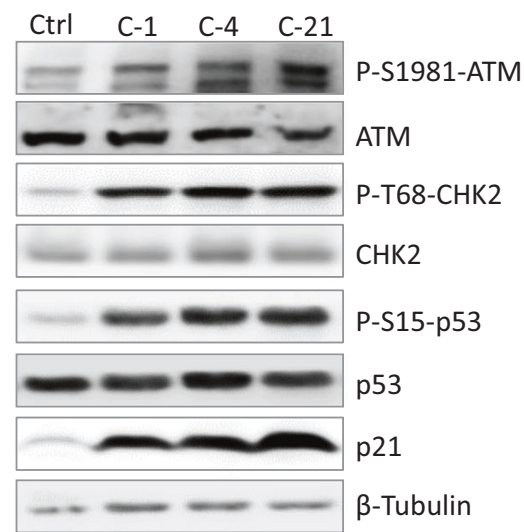

B. GO_DOUBLE_STRAND_BREAK_REPAIR

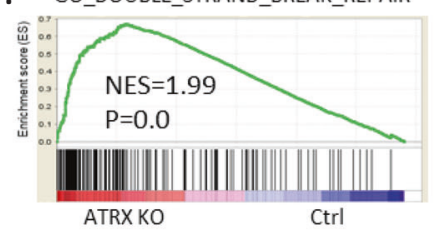

C.

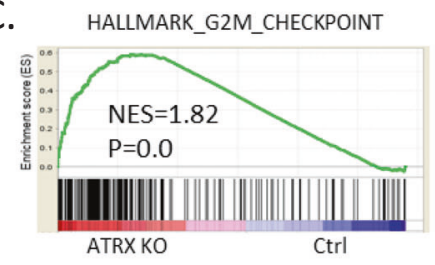

D.

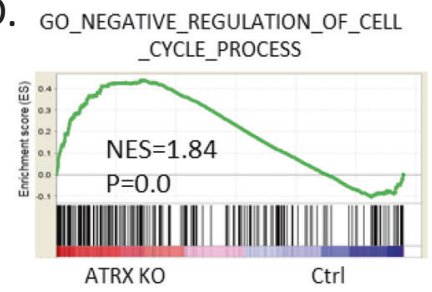

E.

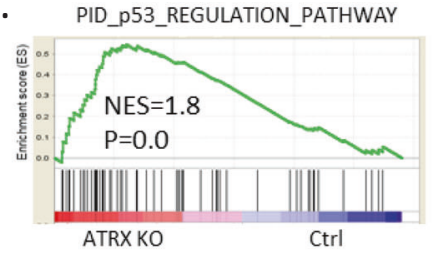

Fig. 2 Increased DNA damage leads to ATM/CHK2/p53 pathway induction in ATRX KO NGP cells. A The Gene Set Enrichment Analysis (GSEA) results of differentially expressed gene sets between Ctrl and ATRX KO NGP cells. Collected nominal p-values and normalized enrichment scores (NES) were listed. B-E GSEA enrichment plot. The gene sets of B 'GO DOUBLE STRAND BREAK REPAIR', C 'HALLMARK G2M CHECKPOINT', D 'GO NEGATIVE REGULATION OF CELL CYCLE PROCESS', and E 'PID p53 REGULATION PATHWAY' were significantly enriched in ATRX KO NGP cells. F Representative immunoblots for the expression of the indicated proteins in cell lysates prepared from Ctrl and ATRX KO NGP cells. ATRX KO NGP cells showed the increased activation of p53-ATM checkpoint proteins. $\beta$-Tubulin was used as a loading control.

Proliferation inhibition by ATRX deficiency may be mediated by increases in DNA damage and activation of the ATM/CHK2/p53 pathway, supporting GSEA results. In response to DNA damage, p53 is phosphorylated by the ATM/CHK2 pathways, leading to cell cycle arrest or apoptosis [36]. Therefore, we investigated the involvement of ATM signaling in response to increased DNA damage. A western blot analysis of Ctrl and ATRX KO NGP cells demonstrated an increase in the activation of ATM in ATRX KO cells (Fig. 2F). ATR activation was not observed in Ctrl or ATRX KO cells (data not shown). Moreover, consistent with the formation of $\mathrm{YH} 2 \mathrm{AX}$ in ATRX KO cells, the activation of ATM-dependent DSB signaling events was also detected in these cells, as revealed by increases in the phosphorylation of CHK2 and p53. Activated p53 then upregulated the expression of p21, which mediated the inhibition of proliferation. Moreover, p53 activation was observed in ATRX KO NB-69 cells, but downstream $\mathrm{YH} 2 \mathrm{AX}$ and p21 only modest (Supplementary Fig. 3C). Collectively, these results revealed that the loss of ATRX in both MYCN-amplified and MYCN single copy TP53 wt NB cells promoted the accumulation of DNA damage and activated the cell cycle checkpoint pathway of DDR, the ATM-CHK2-p53-p21 pathway, leading to cell cycle arrest.

\section{p53 inactivation limits ATRX loss-induced G4 formation and RS in TP53 wt NGP cells}

The inactivation of p53 by a viral oncoprotein or TP53 mutation is commonly observed in ALT-positive cell lines [37]; ALT phenotype 
in NB is associated with ATRX mutation and p53 pathway alterations [20]. To establish whether p53 deficiency with the loss of ATRX promotes ALT in NB cells, a C-terminally V5-tagged dominant-negative p53 (p53_R273H or p53_R175H) vector or control was stably transduced into ATRX KO NGP cells (Fig. 3A, left panel). Mutant p53 exerts a dominant-negative effect by preventing wt p53 from binding to the promoter of its target genes [38]. We initially investigated whether the forced expression of mutant p53 affected the survival of ATRX KO NGP cells. The results obtained showed that it significantly enhanced cell survival during cell cultures, indicating that mutant $\mathrm{p} 53$ reduced the ability of wt p53 to induce cell cycle arrest in ATRX KO NGP cells (Supplementary Fig. 6). The hallmarks associated with ALT were then examined in those cells. Similar to original ATRX KO NGP cells, p53_R273H or p53_R175H did not display C-circles (Supplementary Fig. 7B) or APBs (Supplementary Fig. 7C, D). Moreover, the total telomeric DNA content did not significantly differ between Ctrl and ATRX KO cells with the p53 mutant (Supplementary Fig. 7A). Therefore, NGP cells with ATRX deficiency and the p53 status cannot induce the ALT phenotype, which is associated with ATRX mutation and $\mathrm{p} 53$ pathway aberration in NB.

An investigation of DDR in p53-inactivated ATRX KO NGP cells revealed that the activation of the ATM/CHK2/p53 pathway decreased after p53 inactivation. The levels of key phosphoproteins involved in DDR were lower in ATRX KO NGP cells with the p53 mutant than in original $\mathrm{KO}$ cells (Fig. $3 \mathrm{~A}$, right panel). IF of $\mathrm{YH} 2 \mathrm{AX}$ also showed a weaker signal in p53-inactivated ATRX KO NGP cells (Fig. 3B, C), suggesting the influence of the p53 status on DDR-related cell cycle arrest upon the loss of ATRX. p53 inactivation abolished DDR and also prevented the formation of G4 (Fig. 3D, E) and suppressed the RS pathway in ATRX KO NGP cells (Fig. 3F). Furthermore, IF showed that p53 inactivation reduced phospho-RPA32 foci (Supplementary Fig. 8), consistent with immunoblot results. Therefore, p53 deficiency decreased ATRX loss-induced RS and DDR.

\section{ATRX deficiency-related DDR and RS are not induced in TP53 truncated (C terminus) SK-N-AS cells}

We attempted to confirm the ALT phenotype or p53 deficiencyrelated suppression of RS and DDR upon the loss of ATRX in the NB cell line with mutant TP53 and a MYCN single copy. A previous study reported an ALT phenotype associated with ATRX mutations, the lack of MYCN amplification, and p53 pathway alterations in NB [20]. Therefore, we selected the SK-N-AS cell line carrying a MYCN single copy and TP53 truncation at the C terminus [33]. We isolated several isogenic ATRX KO clones from SK-N-AS cells (discussed in the "Materials and methods" and "Results" sections). We then assessed the ALT status and found that the total telomere content and C-circle levels were below the ALT cut-off value (Supplementary Fig. 9A, B). However, APBs were partially detected in ATRX KO SK-N-AS cells (Supplementary Fig. 9C, D). Although APBs are one of the important markers of the ALT phenotype, C-circles are very specific and quantifiable markers of ALT [39]. Therefore, true ALT features were not observed in SK-N-AS cells after ATRX KO. These results suggest that, at least under the present experimental conditions, the ALT phenotype in NB cell lines was not associated with ATRX mutations, the lack of MYCN amplification, or p53 pathway aberrations.

WST- 8 and colony formation assay results showed that the loss of ATRX did not significantly alter the viability of SK-N-AS cells (Fig. 4A, B). Moreover, $\mathrm{YH} 2 \mathrm{AX}$ foci were not detected in ATRX KO SK-NAS cells, indicating low levels of DNA damage (Fig. 4C) which is contrast with others findings following ATRX KO in TP53 wt SK-N$\mathrm{SH}$ cells with prior p53 inactivation [34]. These relative phenotypic differences may be due to different experimental strategy or cell line specificity. As expected, ATRX deficiency did not induce the ATM/CHK2/p53 pathway (Fig. 4D). In addition, G4 formation was not detected in $\mathrm{KO}$ cells, leading to a suppressed RS response
(Fig. 4E, F). In contrast to previous findings [15], these results indicated that p53 aberrations inhibited ATRX loss-induced RS related to DDR in NB cells.

\section{p53 inactivation leads to the upregulation of G4-resolving helicases and FA pathway proteins involved in replication fork protection}

When replication forks encounter DNA damage, G4 or R-loops may hinder fork progression and increase RS [40]. Under these conditions, specific proteins are recruited to the site of the stalled fork in order to manage the cellular response to this stress. Recent studies described an emerging class of DNA unwinding enzymes, known as helicases, which directly resolve the G4 DNA structure and remove RS during DNA replication $[25,26]$. Another wellstudied G4 helicase regulator or FA pathway protein, FANCD2, stabilizes and helps to restart stalled replication forks, thereby avoiding the generation of DNA damage and genome instability $[27,28]$. To directly investigate how the p53 status affects ATRX loss-mediated RS-induced DSBs, we searched for p53-regulated molecules involved in G4 structure resolution and replication fork protection, leading to the release of RS (Fig. 5A) [30-32]. We speculated that in the absence of ATRX, other G4-resolving helicases or DNA repair molecules may prevent RS during DNA replication. Accordingly, our initial aim was to clarify whether the inactivation of p53 triggered the upregulation of these molecules in Ctrl or ATRX KO NGP cells. Therefore, we compared the mRNA levels of six candidate genes implicated in G4 structure resolution and replication fork recovery in TP53 wt and p53-inactivated Ctrl or ATRX KO NGP cells. Five out of the six genes were upregulated after the inactivation of p53 (RTEL1, FANCD2, BLM, WRN, and RECQL4) (Fig. 5B), which is consistent with previous findings on p53-dependent regulation [30-32]. Importantly, the induction of FANCD2 expression by $\mathrm{p} 53$ inactivation was the strongest. In addition to FANCD2, BLM expression also increased (Fig. 5B). These results appear to support previous findings showing that FANCD2 cooperated with BLM to facilitate stalled fork restart and suppress new origin firing; FANCD2 and BLM were also shown to have cooperative as well as independent roles in the context of stalled fork recovery [41]. Moreover, FANCD2 protein levels were higher in p53-inactivated Ctrl or ATRX KO cells than in TP53 wt Ctrl or ATRX KO NGP cells, which was consistent with qPCR data (Fig. 5B, C). These results support the previous finding of FANCD2 expression being markedly affected by the activation of p53 [30].

We also examined the expression of G4 helicases and FANCD2 at the mRNA level in Ctrl and ATRX KO SK-N-AS cells (Fig. 5D). An immunoblot analysis showed that FANCD2 protein levels remained unchanged between Ctrl or ATRX KO cells (Fig. 5E), suggesting that basal FANCD2 levels in SK-N-AS cells were sufficient for replication fork protection after the loss of ATRX.

Furthermore, ALT-positive NB cell lines with the TP53 mutation (SK-N-FI) had higher FANCD2 and BLM expression levels than TP53 wt (NGP) and TP53-truncated (SK-N-AS) cells (Supplementary Fig. 10A). To date, no functional assays have been performed to establish whether these phenomena are the result of TP53 mutation in ALT-positive NB cells with ATRX LoF. In public datasets (R2, http://r2.amc.nl), FANCD2 expression levels were shown to be strongly associated with an advanced tumor stage and poor prognosis in human NB (Supplementary Fig. 10B, C). These findings suggest that upregulated $F A N C D 2$ expression may promotes the ALT phenotype in ATRX-mutated NB. Collectively, these results indicate that FANCD2 expression levels significantly increased under the setting of p53 deficiency and may cooperate in the protection of stalled replication forks after the loss of ATRX.

p53 deficiency limits ATRX loss-induced RS and genome instability through the FA pathway protein, FANCD2

The loss of ATRX hinders fork stability by G4 or R-loop formation and increases RS (Fig. 1, Supplementary Fig. 3) [11, 17]. Since ATRX 
A.
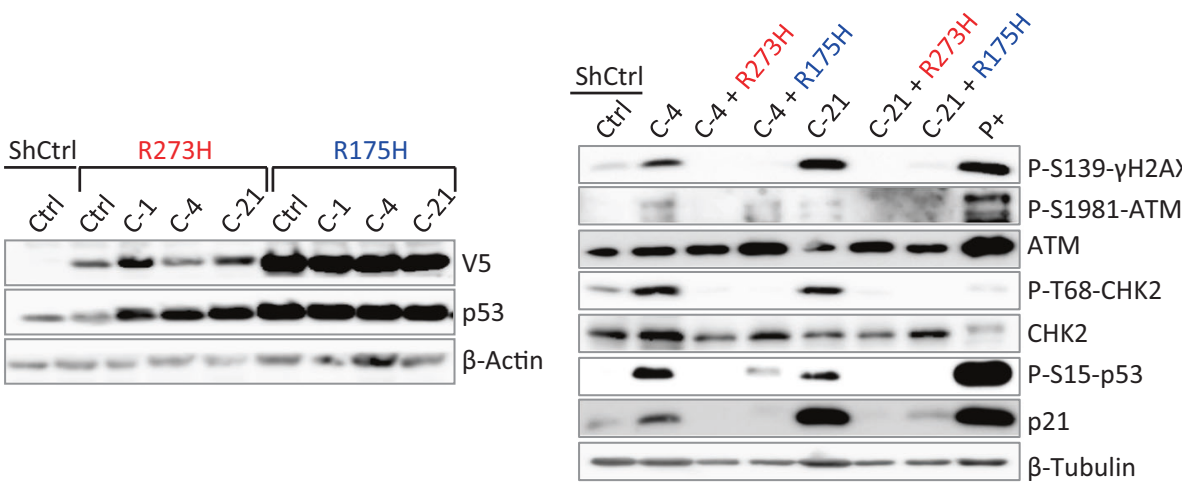

B.
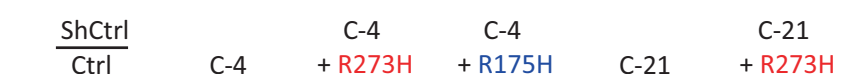

C-21
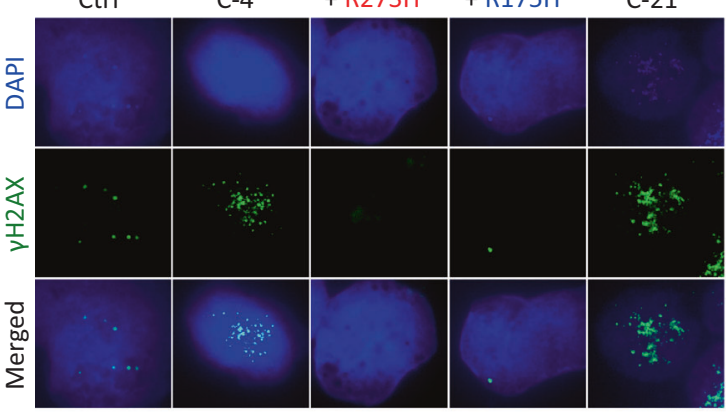

$+\mathrm{R} 273 \mathrm{H}$

$+\mathrm{R} 175 \mathrm{H}$

$\mathrm{P}+$

C.

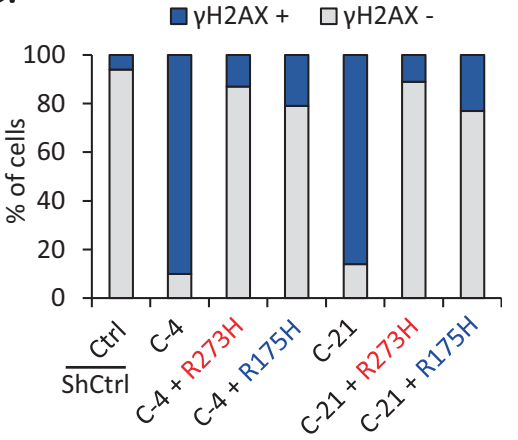

E.

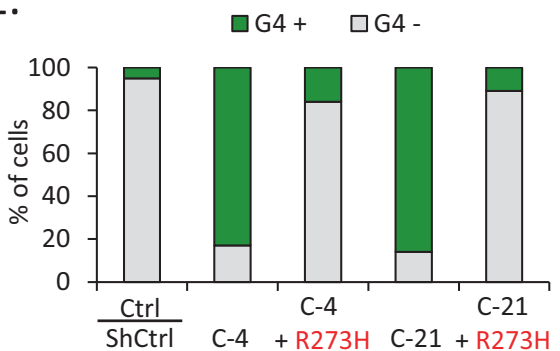

D. $\frac{\text { ShCtrl }}{\text { Ctrl }}$
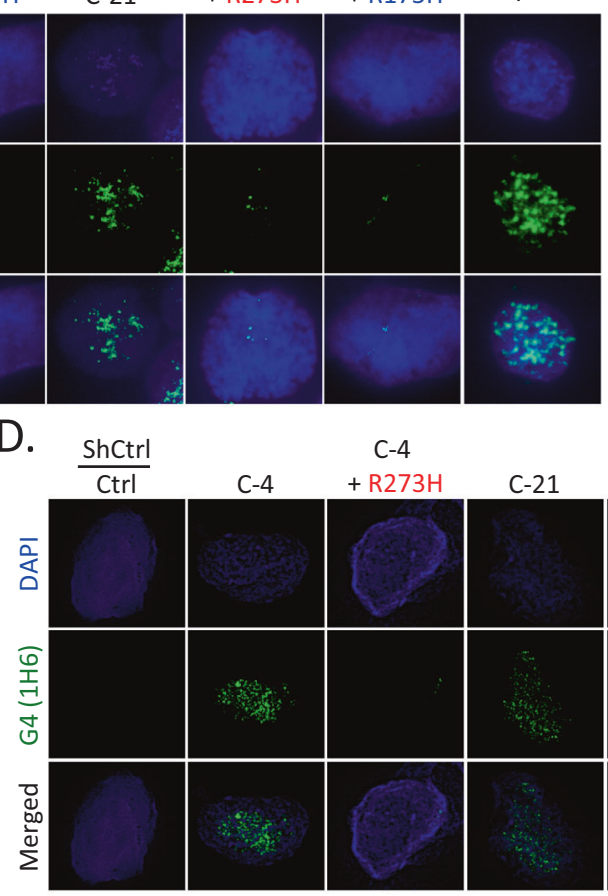

C-21 $+\mathrm{R} 273 \mathrm{H} \quad \mathrm{P}+$

F.

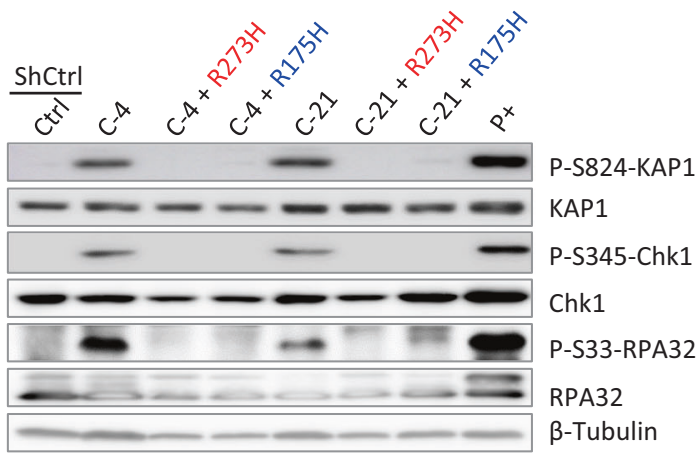

Fig. 3 p53 inactivation limits ATRX loss-induced G4 formation and RS in TP53 wt NGP cells. A Left panel, for p53 inactivation, C-terminally V5-tagged dominant-negative p53 mutants (p53_R273H or p53_R175H) or a control empty vector (ShCtrl) were stably introduced by lentiviral infection into Ctrl or ATRX KO NGP cells. Western blotting using anti-V5 and anti-p53 antibodies analyzed the expression levels of V5-tagged p53 mutants. $\beta$-Actin was used as a loading control. Right panel, immunoblots for the expression of DSB checkpoint proteins in cell lysates prepared from the indicated samples. p53 inactivation in ATRX KO NGP cells results in the loss of the DDR and p53-ATM checkpoint proteins. $\beta$-Tubulin was used as a loading control. As a positive control $(\mathrm{P}+)$, parental NGP cells were treated with doxorubicin $(0.5 \mu \mathrm{g} / \mathrm{mL})$ for $24 \mathrm{~h}$. B, C $\gamma \mathrm{H} 2 \mathrm{AX}$ IF indicates decreased DDR after p53 inactivation in ATRX KO NGP cells. NGP cells treated with doxorubicin (0.5 $\mu \mathrm{g} / \mathrm{mL}, 24 \mathrm{~h})$ were used as the positive control. C Quantification of $\gamma \mathrm{H} 2 \mathrm{AX}+$ cells among 100 cells analyzed in (B). D, E IF staining of G4 (1H6) in the indicated cells. As a positive control ( $\mathrm{P}+$ ), parental NGP cells were treated with the DNA G-quadruplex stabilizer CX-5461 (50 nM) for $24 \mathrm{~h}$ and stained with the anti-G4 (1H6) antibody. Nuclei are counterstained with DAPI (blue). E Quantification of G4+ cells among 100 cells analyzed in (D). F The RS markers p-KAP1, p-Chk1, and p-RPA32 were not induced in p53-inactivated ATRX KO NGP cells, representing a reduction in RS. $\beta$-Tubulin was used as a loading control. 
A.

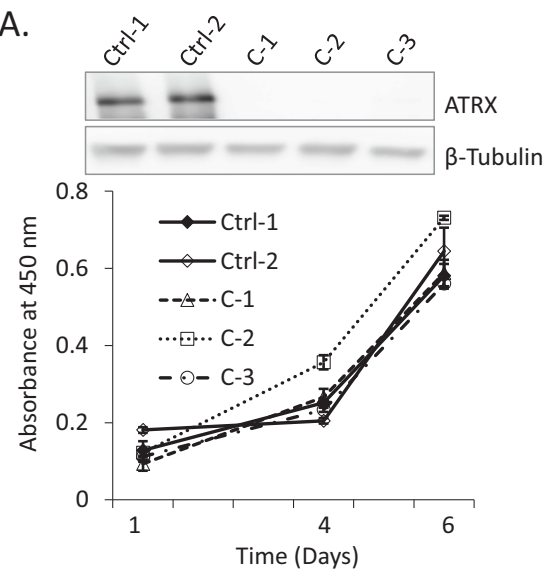

C.
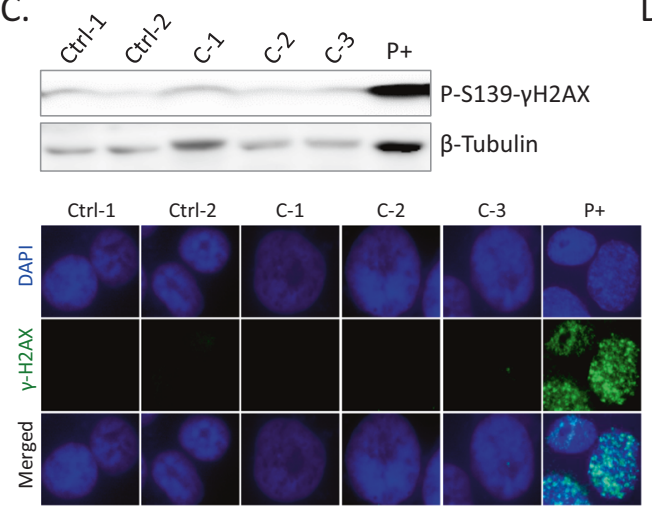

E.

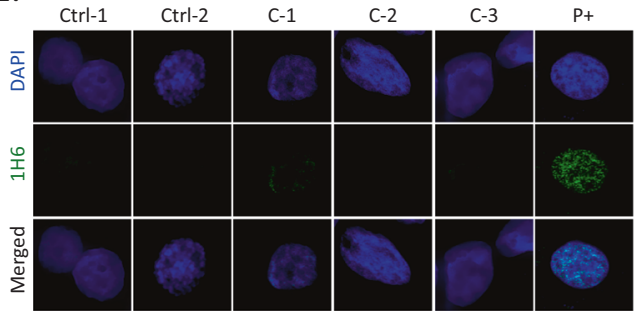

B.

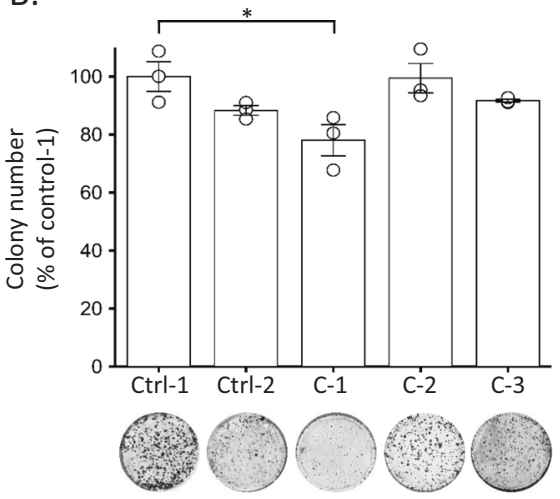

D.

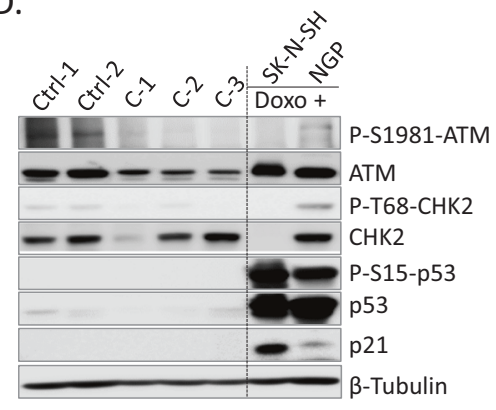

F.

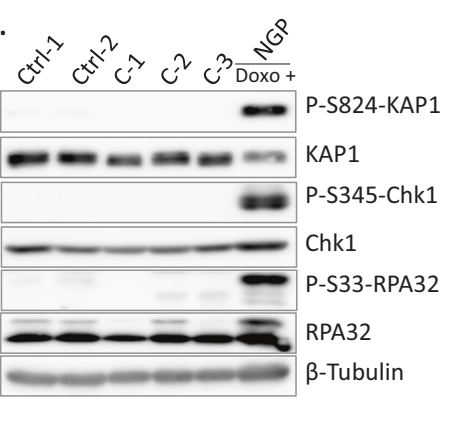

Fig. 4 ATRX deficiency-related DDRs and RS were not induced in TP53-truncated (C terminus) SK-N-AS cells. A Western blot to confirm ATRX ablation in Cas9 control (Ctrl) and ATRX KO (C-1, C-2, and C-3) SK-N-AS cells. Growth curves show that cell viability was unchanged between Ctrl and ATRX KO SK-N-AS cells. Data are expressed as means \pm standard deviation (SD), $N=3$. B Colony formation assays were performed to assess the proliferative abilities of Ctrl and ATRX KO SK-N-AS cells. Lower panel, representative images for clonogenic formation. Error bars represent SD from three technical replicates. ${ }^{*} p<0.05$; A one-way ANOVA with Dunnett's and Tukey's test were used for statistical analyses. C Immunoblot and IF analyses of $\gamma \mathrm{H} 2 \mathrm{AX}$ in ATRX-intact (Ctrl) and ATRX KO SK-N-AS cells with a positive control (parental SK-N-AS cells treated with doxorubicin, $0.5 \mu \mathrm{g} / \mathrm{mL}$ for $24 \mathrm{~h}$ ). D Immunoblots for the expression of p53-ATM checkpoint proteins in cell lysates prepared from Ctrl and ATRX KO SK-N-AS cells. Doxorubicin $(0.5 \mu \mathrm{g} / \mathrm{mL}$ )-treated two NB cell lines (SK-N-SH and NGP) were used as a positive control. E IF staining of G4 (1H6) revealed that ATRX deficiency did not cause G4 formation in ATRX KO SK-N-AS cells. The DNA G-quadruplex stabilizer CX$5461(50 \mathrm{nM}, 24 \mathrm{~h})$ was used as a positive control to stain G4 (1H6). F Whole-cell extracts from Ctrl and ATRX KO SK-N-AS cells were analyzed by western blotting with the p-KAP1 (Ser-824), KAP1, p-Chk1 (Ser-345), Chk1, p-RPA32 (Ser-33), and RPA32 antibodies. $\beta$-Tubulin was used as a loading control. Doxorubicin $(0.5 \mu \mathrm{g} / \mathrm{mL}, 24 \mathrm{~h})$-treated NGP cells were used as a positive control.

and FANCD2 have recently been reported to cooperate in replication fork recovery [29], we investigated whether FANCD2 decreases RS in the context of ATRX deficiency. We validated the knockdown efficiency of several FANCD2 shRNAs in HeLa cells and found that shRNA 3 and shRNA 4 efficiently knocked down FANCD2 at the protein level (Fig. 6A). We then measured the RS marker in two p53-inactivated ATRX KO NGP cells infected with FANCD2 shRNA. In cells lacking FANCD2 and ATRX, we observed elevated levels of $\mathrm{YH} 2 \mathrm{AX}$, which marks DNA damage sites, as well as stronger RS signals (Fig. 6B). Therefore, FANCD2 was required to prevent RS and ultimately genomic instability in ATRX-deficient cells, similar to BRCA2 deficiency $[27,28]$. Cell proliferation assays showed that the inhibition of FANCD2 significantly decreased the survival of p53-inactivated ATRX KO NGP cells (Supplementary Fig. $11 \mathrm{~A}, \mathrm{~B})$. In addition, FANCD2 downregulation reduces the cell viability of ALT-positive SK-N-FI cells (Supplementary Fig. 12A-C). Therefore, FANCD2 may be partly required for the survival of ATRX-deficient cells, suggesting a synthetic lethal relationship between them. Collectively, these results propose a key role for FANCD2 in the protection of replication forks upon the loss of ATRX in NB cells.

\section{DISCUSSION}

In the present study, ATRX deficiency activated RS and DDR through the G4 formation, leading to cell cycle arrest in TP53 wt 
A.

\begin{tabular}{|c|c|c|}
\hline Gene & Function & References \\
\hline RTEL1 & $\begin{array}{l}\text { DNA G4 helicase at telomeres, and disassembly of D-loops and T- } \\
\text { loops and homologous recombination }\end{array}$ & {$[52,53]$} \\
\hline$B L M$ & $\begin{array}{l}\text { Telomeric G4 resolution, replication fork regression, DSB repair and } \\
\text { repair of replication-associated DNA damage }\end{array}$ & {$[26,49,50]$} \\
\hline WRN & Telomeric G4 resolution, replication fork regression and DSB repair & {$[48,51]$} \\
\hline RECQL4 & Cooperates with BLM to unwind DNA G4 & [54] \\
\hline FANCJ & Unwinding of G4 DNA structures, DNA replication and DSB repair & {$[56,57]$} \\
\hline FANCD2 & Replication fork protection and HR directed DNA repair & {$[27,28,55]$} \\
\hline
\end{tabular}

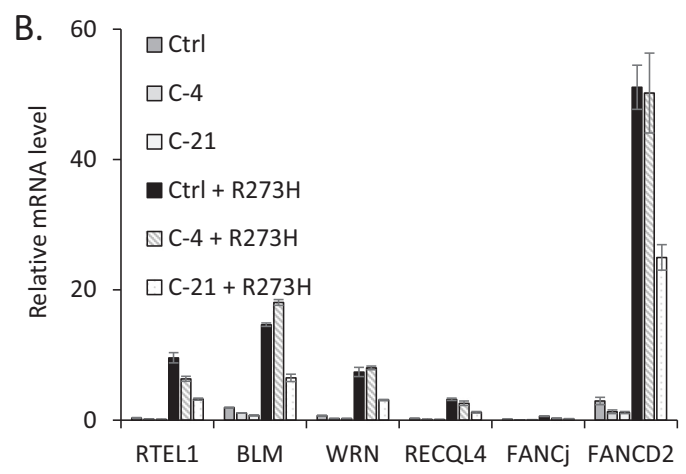

C. $\mathrm{R} 273 \mathrm{H}$

$c^{2} c^{2} c^{2} c^{2} c^{\alpha} c^{2}$

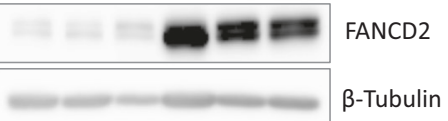

D.

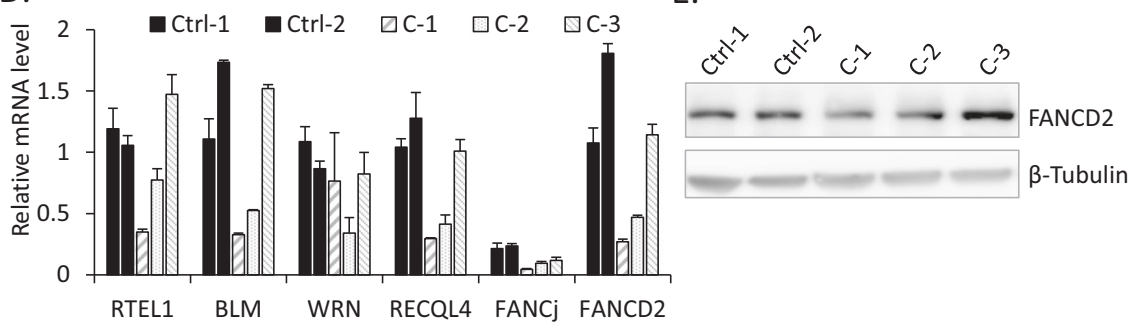

Fig. 5 p53 inactivation leads to the upregulation of G4-resolving helicases and FA pathway proteins involved in replication fork protection. A List of p53 downregulated G4-resolving eukaryotic helicases or FA pathway DNA repair molecules and their proposed biological functions during DNA replication. B A comparison of TP53 wt and p53-inactivated Ctrl or ATRX KO cells suggested the p53-dependent regulation of G4-resolving helicases and the FA pathway DNA repair molecule, FANCD2. Real-time RT-PCR analysis of RTEL1, BLM, WRN, RECQL4, $F A N C J$, and FANCD2 in the indicated samples. Relative expression normalized to GAPDH, mean $\pm \mathrm{SD}(n=3)$. C p53 inactivation leads to increased FANCD2 protein levels. Protein extracts, prepared from TP53 wt and p53-inactivated Ctrl or ATRX KO NGP cells, were immunoblotted with antibodies against FANCD2 and $\beta$-Tubulin. D Real-time RT-PCR analysis of G4-resolving helicase genes and FANCD2 in Ctrl and ATRX KO SK-N-AS cells. Relative expression normalized to GAPDH, mean \pm SD $(n=3)$. E Western blot analysis of FANCD2 in Ctrl and ATRX KO SK-N-AS cells. $\beta$-Tubulin served as a loading control.

NB cells. Both MYCN single and MYCN amplification may activate p53 pathway in ATRX KO cells and drive RS. The synthetic lethal relationship between ATRX mutation and MYCN amplification has been reported [3]. However, TP53-truncated SK-N-AS cells were devoid of the DDR pathway upon the loss of ATRX. Moreover, the inactivation of $\mathrm{p} 53$ by dominant $\mathrm{p} 53$ mutants $(\mathrm{R} 273 \mathrm{H}$ and $\mathrm{R} 175 \mathrm{H})$ in ATRX KO NGP cells rescued cell viability in response to the inhibition of ATM/CHK2/p53 pathway activation. However, the mechanisms by which the p53 status influences the effects of ATRX deficiency on the fate of NB cells currently remain unknown. Therefore, we initially demonstrated that p53 deficiency limited ATRX loss-induced RS/genome integrity in NB cells by regulating G4 helicases and the replication fork-protecting FA pathway protein, FANCD2 (Fig. 6C).

Approximately $24 \%$ of high-risk NB harboring the ALT phenotype and $50-55 \%$ of ALT NB have somatic alterations in ATRX $[1,2,8,9]$. ALT is a HR-based mechanism, and ATRX deficiency induced the formation of G4 in the GC-rich regions of DNA, leading to replication fork stalling and the provision of a substrate for HR [11, 42]. The loss of ATRX also promoted the accumulation of R-loops at telomeres [17], which facilitate the formation of G4 structures in untranscribed DNA strands [43]. Moreover, replication fork stalling and collapse may generate DSBs and DNA damage pathway signaling in ATRX-mutant glioma. While the loss of ATRX induces RS and DNA damage via G4 DNA, ATRX deficiency in conjunction with the chemical stabilization of G4 enhanced RS to induce DNA damage and cell death [11]. Consistent with these findings, we also observed G4 formation, RS, and DDR leading to cell cycle arrest as a consequence of ATRX deficiency in TP53 wt NB cells, supporting the findings that ATRXnegative NPCs induce excessive levels of DNA damage in the embryonic brain caused by DNA RS [15]. Moreover, the ATM/ CHK2/p53 pathway was found to be activated in response to ATRX deficiency-associated DNA damage, indicating that ATRX-null cells 
A.

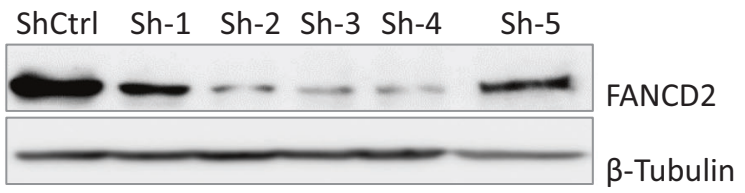

B.

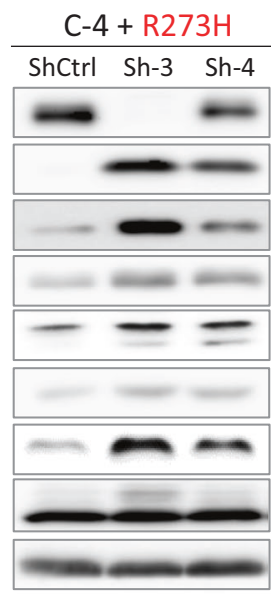
$\mathrm{C}-21+\mathrm{R} 273 \mathrm{H}$

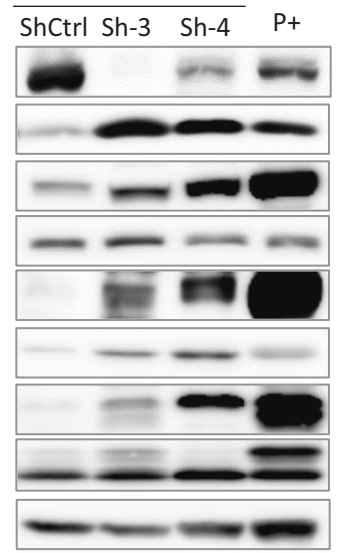

FANCD2

P-S139- $\mathrm{H} 2 \mathrm{AX}$

P-S824-KAP1

KAP1

P-S345-Chk1

Chk1

P-S33-RPA32

RPA32

$\beta$-Tubulin

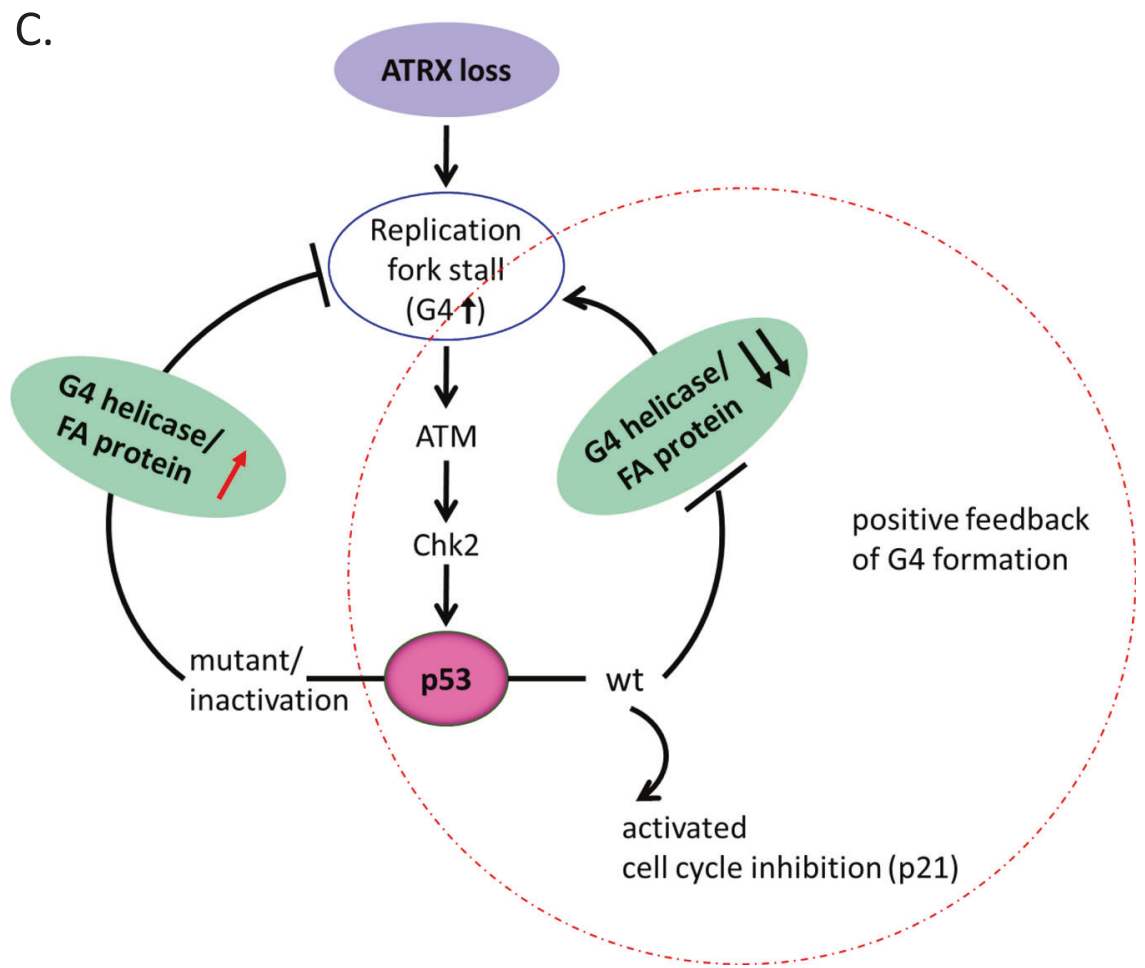

Fig. 6 p53 deficiency limits ATRX loss-induced RS and genome instability through the FA pathway protein, FANCD2. A A western blot analysis showing the silencing efficiency of shRNAs against FANCD2 in HeLa cells. B FANCD2 protein depletion by shRNA was confirmed by immunoblotting. FANCD2 knockdown in p53-inactivated ATRX KO NGP cells resulted in the further activation of the DDR and RS. NGP cells treated with doxorubicin $(0.5 \mu \mathrm{g} / \mathrm{mL}, 24 \mathrm{~h})$ were used as a positive control. C Model for linking ATRX, p53, and replication fork-protecting molecules (G4 helicases or the FA pathway protein, FANCD2) to the DDR, RS, and genomic instability. G4 helicases or FANCD2 activation protected cells from RS in p53-inactivated ATRX-deficient cells. In TP53 wt cells, the loss of ATRX resulted in DNA damage and RS in response to replication fork stalling. p53 inactivation inhibited replication fork stalling by triggering G4-resolving helicases and FANCD2 expression, which may promote G4 structure resolution and replication fork protection.

mostly depend on ATM-associated DDR signaling pathways for DNA repair [15, 44], which is consistent with other findings [45]. p53 inactivation or mutant TP53 with ATRX deficiency inhibited the ATM/CHK2/p53 pathway, which may be attributed to the inhibited formation of G4.
Emerging studies revealed that cancer cells with ALT were hypersensitive to the inhibition of ATR, another component of DNA damage checkpoint-activating kinases other than ATM in human cells [46]. In contrast, in several telomerase-positive and ALT-positive NB or non-NB cell lines treated with an ATR inhibitor, 
ALT-positive cells were not generally more sensitive to ATR inhibition than telomerase-positive cells [34,47]. We also did not observe the significant activation of ATR in NB cells featuring the inactivation of ATRX, which appears to support the finding that differences in ATR inhibitor sensitivity were not related to ATRXdeficient ALT [47].

While p53 inactivation rescued the survival of ATRX-deficient cells, in an attempt to identify the underlying survival mechanism, we focused on $\mathrm{G} 4$ helicases and their regulator, which is involved in the protection of replication forks. We hypothesized that in the absence of ATRX, other molecules may contribute to overcoming the G4 or R-loop secondary DNA structure and promoting stalled replication fork recovery, which are negatively regulated by $p 53$. A number of p53 downregulated G4-interacting helicases that are capable of unfolding G4 structures have been reported [30-32]. They include the RECQ helicase family, WRN, BLM, RECQL4, or RTEL1. WRN and BLM play major roles in unwinding G4 DNA during telomere replication [25, 26, 48-51]; however, RTEL1 has also been implicated in G4 processing and is involved in DNA replication and recombination or required for the maintenance of telomere integrity $[52,53]$. RECQ helicases are strongly expressed in various cancers and their overexpression provides a survival advantage to cancer cells through the protection of stalled replication forks against breakage and possibly the restart of broken replication forks. Therefore, RECQ helicases have been proposed as a target for anticancer therapy because the inhibition of RECQ helicases induced DSBs in different origins and reduced the proliferation of cancer cell lines $[48,54]$. In addition to RECQ helicases, p53 also downregulated FA DNA repair pathway proteins, such as FANCD2 and FANCJ, which are known to be involved in replication fork stabilization or the resolution of R-loops by recruiting RNA processing factors [30, 55] and G4 structures $[48,56,57]$, respectively. Moreover, G4 helicases in complex with FA proteins collaborate in response to stalled replication forks [58]. In our targeted screening, FANCD2, BLM, WRN, RTEL 1, and RECQL4 mRNA expression were increased in ATRX KO NGP cells after the inactivation of p53. FANCD2 and BLM expression was strongly induced by the inactivation of p53. Moreover, we showed that the inhibition of FANCD2 further induced DNA DSB-related RS, thereby decreasing the cellular growth of p53-inactivated ATRX KO NGP and ALT-positive SK-N-FI cells. Consistent with previous findings, FANCD2 in the present study may also appeared to stabilize replication forks [28] and restart forks by cooperating with BLM helicase [41, 58]. FANCD2 also promoted the MRE11 exonuclease-dependent restarting of forks through the deposition of a histone $\mathrm{H} 3$ variant, which is a crucial event during the re-initiation of DNA replication and recruitment of CtIP at stalled replication forks [29].

In contrast, the upregulation of FANCD2 was positively associated with tumor size and a poor prognosis in breast cancer, ovarian cancer, nasopharyngeal carcinoma, glioblastoma, endometrial carcinoma, and esophageal squamous cell carcinoma [59-65]. Similarly, FANCD2 was found to be upregulated in advanced stage NB, and Kaplan-Meier survival curves showed a worse prognosis in patients with high FANCD2 expression levels (R2 database). These findings suggest that the strong expression of FANCD2 may promotes the ALT phenotype in ATRX-mutated NBs, and supports other findings showing that FANCD2 was necessary for telomere maintenance in ALT cells by facilitating the formation of C-circles, possibly by promoting the recruitment of BLM to replication-stressed ALT telomeres [66]. A recent study also described the non-canonical function of FANCD2 with several nuclear receptors to regulate the ALT telomere maintenance pathway [67]. In addition to FANCD2 and $F A N C$, other FA genes, including FANCM, were negatively regulated by $\mathrm{p} 53$ [30]. FANCM is a FA helicase that is essential for the viability of ALT cancer [68]. FANCD2 is required for the functional consequences of FANCM in response to DDR [69]. The inhibition of FANCM was previously shown to induce a potent acute apoptotic phenotype in
ALT cancer cell lines [68], suggesting its potential as an anticancer target in ALT. Therefore, as a regulator of FANCM, FANCD2 will be an excellent target candidate for the treatment of ALT cancers.

The present results demonstrated the dependency of ATRXdeficient cells on p53 dysfunction for cellular survival. Moreover, we proposed that the dependency by these cells on the loss of p53 is mediated by G4 or R-loop resolution and replication fork stability as well as the recovery function of G4 helicases and the FA pathway protein, FANCD2. The present results may also expand the previously suggested roles of FANCD2 as a master regulator/handler of endogenous RS, thereby representing a therapeutic target for ATRX-deficient tumors. Further studies are needed to clarify the mechanisms by which ATRX deficiency interacts with the loss of p53 for NB progression and therapeutic responses.

\section{REFERENCES}

1. Valentijn LJ, Koster J, Zwijnenburg DA, Hasselt NE, van Sluis P, Volckmann R, et al. TERT rearrangements are frequent in neuroblastoma and identify aggressive tumors. Nat Genet. 2015;47:1411-4.

2. Peifer M, Hertwig F, Roels F, Dreidax D, Gartlgruber M, Menon R, et al. Telomerase activation by genomic rearrangements in high-risk neuroblastoma. Nature. 2015;526:700-4.

3. Zeineldin M, Federico S, Chen X, Fan Y, Xu B, Stewart E, et al. MYCN amplification and ATRX mutations are incompatible in neuroblastoma. Nat Commun. 2020;11:913.

4. Watson LA, Goldberg H, Berube NG. Emerging roles of ATRX in cancer. Epigenomics. 2015;7:1365-78.

5. Fishbein L, Khare S, Wubbenhorst B, DeSloover D, D'Andrea K, Merrill S, et al. Whole-exome sequencing identifies somatic ATRX mutations in pheochromocytomas and paragangliomas. Nat Commun. 2015;6:6140.

6. Jiao Y, Shi C, Edil BH, de Wilde RF, Klimstra DS, Maitra A, et al. DAXX/ATRX, MEN1, and $\mathrm{mTOR}$ pathway genes are frequently altered in pancreatic neuroendocrine tumors. Science. 2011;331:1199-203.

7. Chen X, Bahrami A, Pappo A, Easton J, Dalton J, Hedlund E, et al. Recurrent somatic structural variations contribute to tumorigenesis in pediatric osteosarcoma. Cell Rep. 2014;7:104-12.

8. Dagg RA, Pickett HA, Neumann AA, Napier CE, Henson JD, Teber ET, et al. Extensive proliferation of human cancer cells with ever-shorter telomeres. Cell Rep. 2017;19:2544-56.

9. Hartlieb SA, Sieverling L, Nadler-Holly M, Ziehm M, Toprak UH, Herrmann C, et al. Alternative lengthening of telomeres in childhood neuroblastoma from genome to proteome. Nat Commun. 2021;12:1-18.

10. George SL, Parmar V, Lorenzi F, Marshall LV, Jamin Y, Poon E, et al. Novel therapeutic strategies targeting telomere maintenance mechanisms in high-risk neuroblastoma. J Exp Clin Cancer Res. 2020;39:1-10.

11. Wang $Y$, Yang J, Wild AT, Wu WH, Shah R, Danussi $C$, et al. G-quadruplex DNA drives genomic instability and represents a targetable molecular abnormality in ATRX-deficient malignant glioma. Nat Commun. 2019;10:943.

12. Danussi C, Bose P, Parthasarathy PT, Silberman PC, Van Arnam JS, Vitucci M, et al. Atrx inactivation drives disease-defining phenotypes in glioma cells of origin through global epigenomic remodeling. Nat Commun. 2018;9:1-15.

13. Li F, Deng Z, Zhang L, Wu C, Jin Y, Hwang I, et al. ATRX loss induces telomere dysfunction and necessitates induction of alternative lengthening of telomeres during human cell immortalization. EMBO J. 2019;38:1-19.

14. Juhász $S$, Elbakry $A$, Mathes $A$, Löbrich $M$. ATRX promotes DNA repair synthesis and sister chromatid exchange during homologous recombination. Mol Cell. 2018;71:11-24.e7.

15. Watson LA, Solomon LA, Li JR, Jiang Y, Edwards M, Shin-ya K, et al. Atrx deficiency induces telomere dysfunction, endocrine defects, and reduced life span. J Clin Invest. 2013;123:2049-63.

16. Law MJ, Lower KM, Voon HP, Hughes JR, Garrick D, Viprakasit V, et al. ATR-X syndrome protein targets tandem repeats and influences allele-specific expression in a size-dependent manner. Cell. 2010;143:367-78.

17. Nguyen DT, Voon $H$, Xella B, Scott $C$, Clynes D, Babbs $C$, et al. The chromatin remodelling factor ATRX suppresses R-loops in transcribed telomeric repeats. EMBO Rep. 2017;18:914-28.

18. Lewis PW, Elsaesser SJ, Noh KM, Stadler SC, Allis CD. Daxx is an H3.3-specific histone chaperone and cooperates with ATRX in replication-independent chromatin assembly at telomeres. Proc Natl Acad Sci USA. 2010;107:14075-80.

19. Heaphy CM, de Wilde RF, Jiao Y, Klein AP, Edil BH, Shi C. et al. Altered telomeres in tumors with ATRX and DAXX mutations. Science. 2011;333:425 
20. Farooqi AS, Dagg RA, Choi LM, Shay JW, Reynolds CP, Lau LM. Alternative lengthening of telomeres in neuroblastoma cell lines is associated with a lack of MYCN genomic amplification and with p53 pathway aberrations. J Neurooncol. 2014;119:17-26.

21. Schwartzentruber J, Korshunov A, Liu XY, Jones DT, Pfaff E, Jacob K, et al. Driver mutations in histone $\mathrm{H} 3.3$ and chromatin remodelling genes in paediatric glioblastoma. Nature. 2012;482:226-31.

22. Cantero D, Mollejo M, Sepúlveda JM, D'Haene N, Gutiérrez-Guamán MJ, Rodríguez de Lope Á, et al. TP53, ATRX alterations, and low tumor mutation load feature IDH-wildtype giant cell glioblastoma despite exceptional ultra-mutated tumors. Neuro-Oncol Adv. 2020;2:1-11.

23. Seah C, Levy MA, Jiang Y, Mokhtarzada S, Higgs DR, Gibbons RJ, et al. Neuronal death resulting from targeted disruption of the Snf2 protein ATRX is mediated by p53. J Neurosci. 2008;28:12570-80.

24. Benedict $B$, van Harn T, Dekker M, Hermsen S, Kucukosmanoglu A, Pieters W, et al. Loss of p53 suppresses replication-stress-induced DNA breakage in G1/S checkpoint deficient cells. Elife. 2018;7:1-25.

25. Estep KN, Butler TJ, Ding J, Brosh RM. G4-interacting DNA helicases and polymerases: potential therapeutic targets. Curr Med Chem. 2019;26:2881-97.

26. Sauer M, Paeschke K. G-quadruplex unwinding helicases and their function in vivo. Biochem Soc Trans. 2017;45:1173-82.

27. Michl J, Zimmer J, Buffa FM, McDermott U, Tarsounas M. FANCD2 limits replication stress and genome instability in cells lacking BRCA2. Nat Struct Mol Biol. 2016;23:755-7.

28. Kais Z, Rondinelli B, Holmes A, O'Leary C, Kozono D, D'Andrea AD, et al. FANCD2 maintains fork stability in BRCA1/2-deficient tumors and promotes alternative end-joining DNA repair. Cell Rep. 2016;15:2488-99.

29. Raghunandan M, Yeo JE, Walter R, Saito K, Harvey AJ, Ittershagen $S$, et al Functional cross talk between the Fanconi anemia and ATRX/DAXX histone chaperone pathways promotes replication fork recovery. Hum Mol Genet. 2020;29:1083-95.

30. Jaber S, Toufektchan E, Lejour V, Bardot B, Toledo F. p53 downregulates the Fanconi anaemia DNA repair pathway. Nat Commun. 2016;7:11091.

31. Simeonova I, Jaber S, Draskovic I, Bardot B, Fang M, Bouarich-Bourimi R, et al. Mutant mice lacking the p53 C-terminal domain model telomere syndromes. Cell Rep. 2013;3:2046-58.

32. Toufektchan $E$, Toledo $F$. The guardian of the genome revisited: $p 53$ downregulates genes required for telomere maintenance, DNA repair, and centromere structure. Cancers. 2018;10:135.

33. Nakamura $Y$, Ozaki T, Niizuma $H$, Ohira $M$, Kamijo $T$, Nakagawara A. Functional characterization of a new p53 mutant generated by homozygous deletion in a neuroblastoma cell line. Biochem Biophys Res Commun. 2007;354:892-8.

34. George SL, Lorenzi F, King D, Hartlieb S, Campbell J, Pemberton H, et al. Therapeutic vulnerabilities in the DNA damage response for the treatment of ATRX mutant neuroblastoma. EBioMedicine. 2020;59:102971.

35. Zhang M, Wang B, Li T, Liu R, Xiao Y, Geng X, et al. Mammalian CST averts replication failure by preventing $G$-quadruplex accumulation. Nucleic Acids Res. 2019;47:5243-59.

36. Lanz MC, Dibitetto D, Smolka MB. DNA damage kinase signaling: checkpoint and repair at 30 years. EMBO J. 2019;38:e101801.

37. Henson JD, Reddel RR. Assaying and investigating Alternative Lengthening of Telomeres activity in human cells and cancers. FEBS Lett. 2010;584:3800-11.

38. Willis A, Jung EJ, Wakefield $T$, Chen X. Mutant p53 exerts a dominant negative effect by preventing wild-type $\mathrm{p} 53$ from binding to the promoter of its target genes. Oncogene. 2004;23:2330-8.

39. Henson JD, Cao Y, Huschtscha LI, Chang AC, Au AY, Pickett HA, et al. DNA $\mathrm{C}$-circles are specific and quantifiable markers of alternative- lengthening-oftelomeres activity. Nat Biotechnol. 2009;27:1181-5.

40. Maffia A, Ranise C, Sabbioneda S. From R-loops to G-quadruplexes: emerging new threats for the replication fork. Int J Mol Sci. 2020;21:1506.

41. Chaudhury I, Sareen A, Raghunandan M, Sobeck A. FANCD2 regulates BLM complex functions independently of $\mathrm{FANCl}$ to promote replication fork recovery. Nucleic Acids Res. 2013;41:6444-59.

42. Clynes D, Jelinska C, Xella B, Ayyub H, Scott C, Mitson M, et al. Suppression of the alternative lengthening of telomere pathway by the chromatin remodelling factor ATRX. Nat Commun. 2015;6:7538.

43. Lee CY, McNerney C, Ma K, Zhao W, Wang A, Myong S. R-loop induced G-quadruplex in non-template promotes transcription by successive R-loop formation. Nat Commun. 2020;11:1-15.

44. Huh MS, Ivanochko D, Hashem LE, Curtin M, Delorme M, Goodall E, et al. Stalled replication forks within heterochromatin require ATRX for protection. Cell Death Dis. 2016;7:e2220-12.

45. Conte D, Huh M, Goodall E, Delorme M, Parks RJ, Picketts DJ. Loss of Atrx sensitizes cells to DNA damaging agents through p53-mediated death pathways. PLoS ONE. 2012;7:1-9.
46. Flynn RL, Cox KE, Jeitany M, Wakimoto H, Bryll AR, Ganem NJ, et al. Alternative lengthening of telomeres renders cancer cells hypersensitive to ATR inhibitors. Science. 2015;347:273-7.

47. Deeg Kl, Chung I, Bauer C, Rippe K. Cancer cells with alternative lengthening of telomeres do not display a general hypersensitivity to ATR inhibition. Front Oncol. 2016:6:1-7.

48. Maestroni L, Matmati S, Coulon S. Solving the telomere replication problem Genes. 2017;8:55

49. Sun H, Karow JK, Hickson ID, Maizels N. The Bloom's syndrome helicase unwinds G4 DNA. J Biol Chem. 1998;273:27587-92.

50. Drosopoulos WC, Kosiyatrakul ST, Schildkraut CL. BLM helicase facilitates telomere replication during leading strand synthesis of telomeres. J Cell Biol. 2015;210:191-208.

51. Crabbe L. Defective telomere lagging strand synthesis in cells lacking WRN helicase activity. Science. 2004;306:1951-3.

52. Vannier JB, Sarek G, Boulton SJ. RTEL1: functions of a disease-associated helicase. Trends Cell Biol. 2014;24:416-25.

53. Vannier JB, Pavicic-Kaltenbrunner V, Petalcorin MIR, Ding H, Boulton SJ. RTEL1 dismantles $\mathrm{T}$ loops and counteracts telomeric G4-DNA to maintain telomere integrity. Cell. 2012;149:795-806.

54. Singh DK, Popuri V, Kulikowicz T, Shevelev I, Ghosh AK, Ramamoorthy M, et al The human RecQ helicases BLM and RECQL4 cooperate to preserve genome stability. Nucleic Acids Res. 2012;40:6632-48.

55. Okamoto $\mathrm{Y}$, Abe $\mathrm{M}$, Itaya $\mathrm{A}$, Tomida J, Ishiai $\mathrm{M}$, Takaori-Kondo $\mathrm{A}$, et al. FANCD2 protects genome stability by recruiting RNA processing enzymes to resolve R-loops during mild replication stress. FEBS J. 2019;286:139-50.

56. Wu Y, Shin-ya K, Brosh RM. FANCJ helicase defective in Franconia anemia and breast cancer unwinds G-quadruplex DNA to defend genomic stability. Mol Cell Biol. 2008;28:4116-28.

57. London TB, Barber L, Mosedale G, Kelly GP, Balasubramanian S, Hickson ID, et al. FANCJ is a structure-specific DNA helicase associated with the maintenance of genomic G/C tracts. J Biol Chem. 2008;283:36132-9.

58. Datta A, Brosh RM Jr. Holding all the cards-how fanconi anemia proteins deal with replication stress and preserve genomic stability. Genes. 2019;10:170.

59. Rudland PS, Platt-Higgins AM, Davies LM, de Silva Rudland S, Wilson JB, Aladwani A, et al. Significance of the Fanconi anemia FANCD2 protein in sporadic and metastatic human breast cancer. Am J Pathol. 2010;176:2935-47.

60. Feng $L$, Jin F. Expression and prognostic significance of Fanconi anemia group D2 protein and breast cancer type 1 susceptibility protein in familial and sporadic breast cancer. Oncol Lett. 2019;17:3687-3700.

61. Moes-Sosnowska J, Rzepecka IK, Chodzynska J, Dansonka-Mieszkowska A, Szafron LM, Balabas A, et al. Clinical importance of FANCD2, BRIP1, BRCA1, BRCA2 and FANCF expression in ovarian carcinomas. Cancer Biol Ther. 2019;20:843-54

62. Xu S, Zhao F, Liang $Z$, Feng $H$, Bao $Y, X u$ W, et al. Expression of FANCD2 is associated with prognosis in patients with nasopharyngeal carcinoma. Int J Clin Exp Pathol. 2019;12:3465-73.

63. Patil $A A$, Sayal $P$, Depondt $M L$, Beveridge RD, Roylance A, Kriplani $D H$, et al FANCD2 re-expression is associated with glioma grade and chemical inhibition of the Fanconi Anaemia pathway sensitises gliomas to chemotherapeutic agents. Oncotarget. 2014;5:6414-24.

64. Mhawech-Fauceglia P, Wang D, Kim G, Sharifian M, Chen X, Liu Q, et al. Expression of DNA repair proteins in endometrial cancer predicts disease outcome. Gynecol Oncol. 2014;132:593-8.

65. Lei LC, Yu VZ, Ko JMY, Ning L, Lung ML. Fancd2 confers a malignant phenotype in esophageal squamous cell carcinoma by regulating cell cycle progression. Cancers. 2020;12:1-14.

66. Pan X, Chen Y, Biju B, Ahmed N, Kong J, Goldenberg M, et al. FANCM suppresses DNA replication stress at ALT telomeres by disrupting TERRA R-loops. Sci Rep. 2019;9:1-14.

67. Xu M, Qin J, Wang L, Lee HJ, Kao CY, Liu D, et al. Nuclear receptors regulate alternative lengthening of telomeres through a novel noncanonical FANCD2 pathway. Sci Adv. 2019;5:1-16.

68. Silva B, Pentz R, Figueira AM, Arora R, Lee YW, Hodson C, et al. FANCM limits ALT activity by restricting telomeric replication stress induced by deregulated BLM and R-loops. Nat Commun. 2019;10:1-16.

69. Huang J, Zhang J, Bellani MA, Pokharel D, Gichimu J, James RC, et al. Remodeling of interstrand crosslink proximal replisomes is dependent on ATR, FANCM, and FANCD2. Cell Rep. 2019;27:1794-808.e5.

\section{ACKNOWLEDGEMENTS}

We thank Mr. Daniel Mrozek (Medical English Service, Kyoto) for his English editorial assistance. 
12

\section{AUTHOR CONTRIBUTIONS}

Conceptualization: TK and JA. Data curation: JA, YK, and PS. Formal analysis: PS, HT, MH, RPS, KM, SS, TW, KA, and TK. Resources: TK, KA, and JA. Analyzed data: JA, YK, PS, MO, RPS, and TK. Validation: JA, YK, PS, MO, and TK. Writing, reviewing \& editing: JA and TK. Study supervision: TK. Project administration: TK. Funding acquisition: TK and JA.

\section{FUNDING}

This work was supported by JSPS KAKENHI Grant Number $18 \mathrm{~K} 15256$ and a JSPS KAKENHI Grant-in-Aid for Scientific Research (B) (19H03625).

\section{COMPETING INTERESTS}

The authors declare no competing interests.

\section{ADDITIONAL INFORMATION}

Supplementary information The online version contains supplementary material available at https://doi.org/10.1038/s41389-021-00363-6.

Correspondence and requests for materials should be addressed to Takehiko Kamijo.
Reprints and permission information is available at http://www.nature.com/ reprints

Publisher's note Springer Nature remains neutral with regard to jurisdictional claims in published maps and institutional affiliations. Attribution 4.0 International License, which permits use, sharing, adaptation, distribution and reproduction in any medium or format, as long as you give appropriate credit to the original author(s) and the source, provide a link to the Creative Commons license, and indicate if changes were made. The images or other third party material in this article are included in the article's Creative Commons license, unless indicated otherwise in a credit line to the material. If material is not included in the article's Creative Commons license and your intended use is not permitted by statutory regulation or exceeds the permitted use, you will need to obtain permission directly from the copyright holder. To view a copy of this license, visit http://creativecommons. org/licenses/by/4.0/.

(c) The Author(s) 2021 